\title{
Numerical studies on Feldkamp-type and Katsevich-type algorithms for cone-beam scanning along nonstandard spirals
}

\author{
Jiehua Zhu ${ }^{\mathrm{a}, \mathrm{b}}$, Shiying Zhao ${ }^{\mathrm{a}}$, Hengyong Yuc ${ }^{\mathrm{c}}$, Yangbo Ye, ${ }^{\mathrm{a}, \mathrm{b}}$, Seung Wook Lee ${ }^{\mathrm{d}}$, *Ge Wang ${ }^{\mathrm{a}, \mathrm{b}}$ \\ ${ }^{\mathrm{a}}$ CT/Micro-CT Lab, Department of Radiology, ${ }^{\mathrm{b}}$ Department of Mathematics, \\ University of Iowa, Iowa City, IA52242, USA; \\ ${ }^{\mathrm{c} C o l l e g e}$ of Communication Engineering, Hangzhou Dianzi University, Zhejiang 310018, China; \\ ${ }^{\mathrm{d}}$ Korea Atomic Energy Research Institute, Daejeon 305600, South Korea
}

\begin{abstract}
In this paper, we perform numerical studies on Feldkamp-type and Katsevich-type algorithms for cone-beam reconstruction with a nonstandard spiral locus to develop an electron-beam micro-CT scanner. Numerical results are obtained using both the approximate and exact algorithms in terms of image quality. It is observed that the two algorithms produce similar quality if the cone angle is not large and/or there is no sharp density change along the $z$ direction. The Katsevich-type algorithm is generally preferred due to its nature of exactness.
\end{abstract}

Keywords: Computed tomography (CT), cone-beam, nonstandard spiral scanning, Feldkamp-type algorithm, Katsevisch-type algorithm.

\section{INTRODUCTION}

Electron beam computed tomography (EBCT), also known as ultra-fast computed tomography, is a CT scanner specifically developed for imaging of the heart. In the EBCT design, an electron beam is focused magnetically directed at the tungsten target. Then, the target yields a focus spot generating X-ray photons. These photons penetrate a patient, and are detected by an array of detectors for use in tomographic reconstruction. EBCT can very accurately identify and quantify the calcium deposits in or around coronary arteries, which is an indication of coronary heart diseases. However, there are two major weaknesses with the current EBCT techniques. First, it is not in cone-beam geometry and does not support spiral/helical scanning, while it has been recognized that spiral/helical cone-beam scanning is advantageous for the next generation of biomedical CT. Second, the X-ray spot is not intensive sufficiently to produce the image quality the mechanical rotation based scanners can achieve. Recently Wang and $\mathrm{Ye}^{1}$ proposed to upgrade the EBCT design with a spiral cone-beam scanning capability and adapt the architecture for small animal imaging, especially for cardiac studies of the mouse. This electron-beam micro-CT (EBMCT) prototype is intended to improve the current temporal resolution of micro-CT by an order of magnitude, allowing more than 10 tomographic reconstructions per cardiac cycle of the mouse with adequate image quality. Non-standard spiral cone-beam reconstruction algorithms, of both approximate and exact types, are of our interest for EBMCT.

In the standard spiral cone-beam image reconstruction, both exact and approximate algorithms have their relative merits in terms of image quality ${ }^{2}$. Ideally, the exact algorithm reconstructs an image accurately. Practically, the approximate algorithm often produces satisfactory results, and is computationally efficient. Therefore, both types of the algorithms have been extensively studied over past two decades. In 1984, Feldkamp et al. ${ }^{3}$ proposed an approximate cone-beam algorithm for circular cone-beam scanning. The Feldkamp algorithm has proved to be quite excellent, especially for a small cone angle. In 1991, Wang et al. ${ }^{4}$ generalized the Feldkamp algorithm for cone-beam scanning along various scanning loci, which works well for spherical, rod-like or plate-shaped objects with various scanning loci including nonstandard spirals.

In 2001, Katsevich ${ }^{5}$ derived the first theoretically exact reconstruction formula for the spiral cone-beam geometry in the filtered backprojection format. The work by Katsevich is based on the PI-line and Tam-Danielsson detection geometry. For our purpose of EBMCT, we studied PI-line geometry and generalized the Tam-Danielsson window in the nonstandard spiral case of constant radius but variable pitch $^{6}$ and constant pitch but variable radii ${ }^{7}$. Recently, we proposed a Katsevich-type algorithm for variable radius spiral scanning ${ }^{8}$. 
In this paper, we perform a numerical study on Feldkamp-type and Katsevich-type algorithms for cone-beam scanning along a nonstandard spiral of constant pitch and variable radius. The paper is organized as follows. A generalized Feldkamp algorithm and a generalized Katsevich algorithm are briefly summarized in Sections 2 and 3, respectively. Numerical simulation results obtained using the two algorithms are presented in Section 4. The related issues are discussed in the last section.

\section{GENERALIZED FELDKAMP ALGORITHM}

Let

$$
\vec{y}(s)=(R(s) \cos s, R(s) \sin s, h(s)), \quad a \leq s \leq b,
$$

be a nonstandard spiral, where $s$ represents the rotation angle, $h^{\prime}(s)>0$ for any $s$ in $[a, b]$ so that $h(s)$ is always increasing. In a 3-D coordinate system $(x, y, z)$, it is assumed that an object is centered at the coordinate system origin and its characteristic function $f(x, y, z)$ is confined by a cylinder $U$ of radius $\bar{R}$, that is, $f(x, y, z)=0$ if $\sqrt{x^{2}+y^{2}}>\bar{R}$. The problem is then to reconstruct the function $f(x, y, z)$ from the X-ray projection images.

The generalized Feldkamp reconstruction ${ }^{4}$ is performed based on the following formula:

$$
g(x, y, z)=\frac{1}{2} \int_{0}^{2 \pi} \frac{R(s)^{2}}{(R(s)-v)^{2}} \int_{-\infty}^{\infty} R_{s}(p, \xi) f\left(\frac{R(s) u}{R(s)-v}-p\right) \frac{R(s)}{\sqrt{R(s)^{2}+p^{2}+\xi^{2}}} d p d s,
$$

where $(u, v)$ denotes the rotated coordinate system described by

$$
\left\{\begin{array}{l}
u=x \cos s+y \sin s \\
v=-x \sin s+y \cos s
\end{array},\right.
$$

$R_{s}(p, \xi)$ the two-dimensional equi-spatial cone-beam projection data, $f(\cdot)$ a reconstruction filter,

$$
\xi=\frac{R(s) \tilde{z}(s)}{R(s)-s},
$$

and the local coordinate system $[x, y, \tilde{z}(s)]$ associated with the angle $s$ is defined by

$$
\tilde{z}(s)=z-h(s) \text {. }
$$

The generalized algorithm (2) allows various scanning loci. Here we focus on the nonstandard spiral scanning with variable radius but constant pitch. Basically, the following three steps are followed to reconstruct a $z$-slice: collecting data from a spiral turn centered on the $z$-slice, filtering the data, and backprojecting the filtered data onto the $z$-slice.

\section{GENERALIZED KATSEVICH ALGORITHM}

Assume that the scanning locus satisfies the conditions for the uniqueness of the PI line in the case of variable radius spiral scanning ${ }^{7}$, the Katsevich algorithm can be generalized for variable radius cone-beam scanning ${ }^{8}$. The generalized Katsevich algorithm consists of the following steps:

- Step 1: Compute the first derivative of cone-beam data with respect to variable $s$ for a fixed direction pointing to $\vec{x} \in U$ from a source $\vec{y}(s)$ on the scanning locus (1).

- Step 2: Determine the PI-segment, whose endpoints are denoted by $s_{b}(\vec{x})$ and $s_{t}(\vec{x}), \vec{x} \in U$, by first considering a family of the PI lines intersecting the vertical line $V$ which contains $\vec{x}$, and then numerically locating the PI line that intersects the line $V$ and $\vec{y}\left(s_{t}\right)$ with $s_{t} \in\left(s_{b}, s_{b}+2 \pi\right)$.

- Step 3: Perform slant filtering of the first derivative data.

- Step 4: Backproject the filtered data according to weights inversely proportional to the distance from $\vec{x}$ to the source $\vec{y}(s)$ to reconstruct $f(\vec{x})$. 


\section{COMPUTER SIMULATION}

\subsection{Imaging geometries and phantoms}

We selected spirals with monotonically increasing $R(s)$ and constant pitch:

$$
\vec{y}(s)=(R(s) \cos s, R(s) \sin s, h(s)), R(s)=a s+b, h(s)=\frac{h_{p} s}{2 \pi} .
$$

Note that the Archimedean spiral $R(s)=a s+b$ satisfies the PI-line uniqueness conditions ${ }^{7}$. The simulation parameters were designed according to the preliminary specifications of our EBMCT system as listed in Table 1. The scanning locus was then specified as $R(s)=\frac{25}{6 \pi} s+\frac{85}{2}, h_{p}=25 \mathrm{~mm}$.

Table 1. Preliminary specifications of the electron-beam micro-CT prototype for small animal cardiac imaging.

\begin{tabular}{||l|l||l|l||}
\hline \hline Field of view & $30-50 \mathrm{~mm}$ & Number of views per rotation & $30-100$ \\
\hline Source to axis distance & $30-60 \mathrm{~mm}$ & Spatial \& contrast resolution & $\sim 100 \mu \mathrm{m}, \sim 5 \%$ \\
\hline Source to detector distance & $125-150 \mathrm{~mm}$ & Scanning speed & $\sim 50 \mathrm{HZ}$ \\
\hline
\end{tabular}

As shown in Fig. 1, the radius of mouse was taken to be $\bar{R}=20 \mathrm{~mm}$, the length of its thorax was $L=50 \mathrm{~mm}$, the detector plate was $D=95 \mathrm{~mm}$ away from the center axis of the mouse. Because the generalized Feldkamp algorithm requires that all the points in the field of view could be seen from the corresponding spiral turn, we can easily determine the minimum detector size. Suppose that the center of the thorax is placed at the origin, as shown in Fig. 2(a), to make the bottom slice $z_{b o t}=-25 \mathrm{~mm}$ visible from the starting point $s_{1}=-3 \pi$ on the lower half turn, the width and height of the detector plate shall be respectively

$$
W=\frac{2 \bar{R}\left(D+R\left(s_{1}\right)\right)}{\sqrt{R^{2}\left(s_{1}\right)-\bar{R}^{2}}}=224 \mathrm{~mm}, H=\frac{h\left(D+R\left(s_{1}\right)\right)}{R\left(s_{1}\right)-\bar{R}}=313 \mathrm{~mm} .
$$

To let this slice visible from the ending point of the upper half turn, $s=-\pi$, we need $W=165 \mathrm{~mm}$ and $H=185 \mathrm{~mm}$ respectively. Then, we can consider the top slice $z_{\text {top }}=25$ similarly. It is straightforward to obtain the upper-half-turn values $W=117 \mathrm{~mm}$ and $H=107 \mathrm{~mm}$, and the lower-half-turn values $W=134 \mathrm{~mm}$ and $H=132 \mathrm{~mm}$ in the top-slice case. Therefore, the minimum detector plate should be $224 \mathrm{~mm}$ by $313 \mathrm{~mm}$ as required by the generalized Feldkamp algorithm. Other half-scan or PI-line based Feldkamp-type algorithms were not included in this preliminary work.

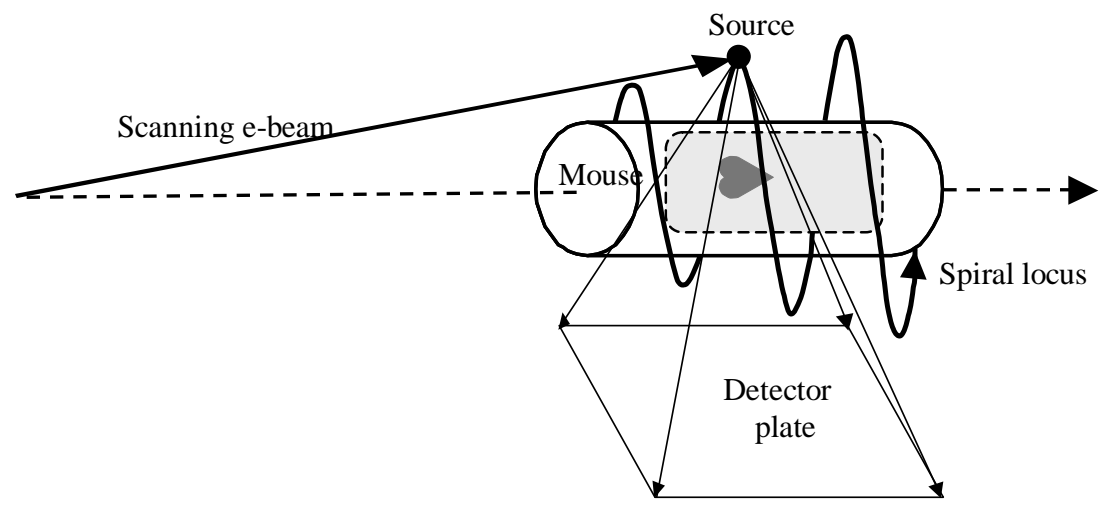

Fig. 1.Electron-beam micro-CT using variable radius spiral cone-beam scanning. 


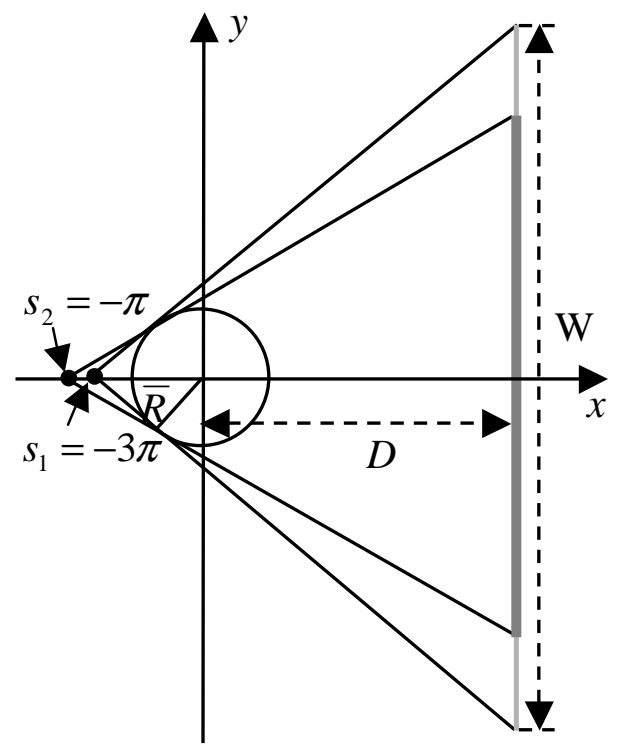

(a)

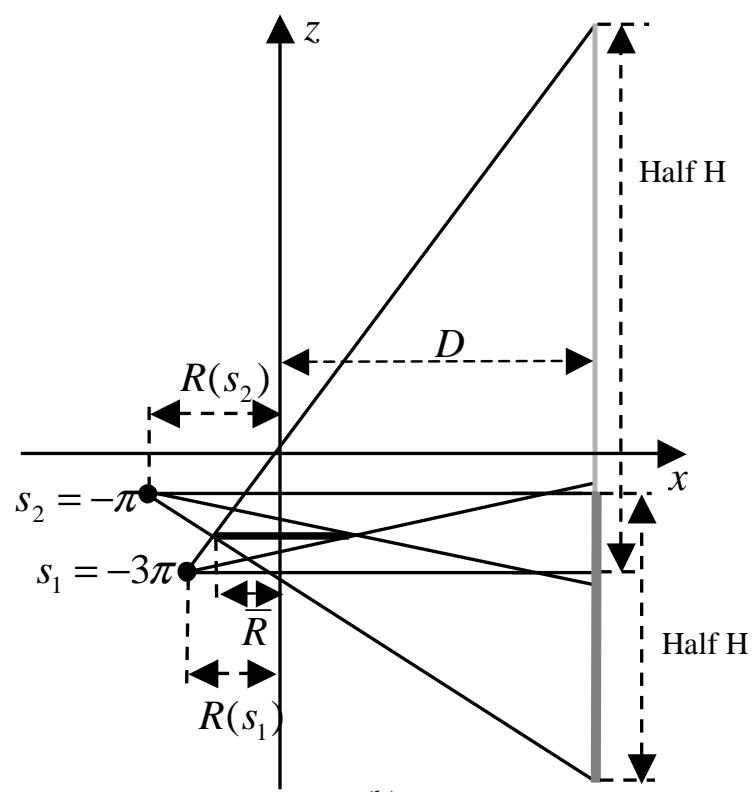

(b)

Fig. 2. Projective views of the imaging geometry for the bottom slice $z=-25 \mathrm{~mm}$. (a) Top view for the computation of the detector width, (b) side view for the computation of the detector height.

In the generalized Katsevich algorithm, the minimum detection window is the region in the detector plate bounded by the cone-beam projections of the upper and lower turns of the nonstandard 3D spiral locus starting from a given source position. We deduced the following boundary equations of the minimum detection window for the source position $\vec{y}\left(s_{0}\right)^{7}$,

$$
d_{1}=\frac{R(s)\left(R+R\left(s_{0}\right)\right) \sin \left(s-s_{0}\right)}{R\left(s_{0}\right)-R(s) \cos \left(s-s_{0}\right)}, d_{2}=\frac{\left(R+R\left(s_{0}\right)\right)\left(s-s_{0}\right) h}{R\left(s_{0}\right)-R(s) \cos \left(s-s_{0}\right)} .
$$

Because the smallest scanning radius leads to the largest cone angle and the greatest detection window, we can simply compute the size of the detector plate for the source with the smallest scanning radius. In this way, we have the width $224 \mathrm{~mm}$ and the height $138 \mathrm{~mm}$.

In our simulation, the parameters were normalized to unit relative to the object radius in the cases of the Shepp-Logan phantom and the disk phantom. The imaging geometries for both the algorithms are given in Table 2.

Table 2. Nonstandard cone-beam scanning parameters used in the numerical simulation.

\begin{tabular}{|c|c|c|}
\hline & GENERALIZED FELDKAMP & GENERALIZED KATSEVICH \\
\hline Scanning radius & $R(s)=\frac{25}{6 \pi} s+\frac{85}{2}$ & $R(s)=\frac{25}{6 \pi} s+\frac{85}{2}$ \\
\hline Helical pitch & $25 \mathrm{~mm}$ & $25 \mathrm{~mm}$ \\
\hline Object radius & $20 \mathrm{~mm}$ & $20 \mathrm{~mm}$ \\
\hline Origin to detector distance & $95 \mathrm{~mm}$ & $95 \mathrm{~mm}$ \\
\hline Number of views per rotation & 100 & 100 \\
\hline Detector size & $224 \mathrm{~mm}$ by $313 \mathrm{~mm}$ & 213 \\
\hline Number of detector rows & 482 & 0.65 \\
\hline Horizontal detector sampling interval & 0.65 & 346 \\
\hline Number of detector columns & 346 & 0.65 \\
\hline Vertical detector sampling interval & 0.65 & $256 \times 256 \times 256$ \\
\hline Reconstruction matrix & $256 \times 256 \times 256$ & by $138 \mathrm{~mm}$ \\
\hline
\end{tabular}


The 3D Shepp-Logan phantom ${ }^{9}$ consists of 10 ellipsoids. The effective X-ray absorption coefficient at a point is the sum of the relative parameters of the ellipsoids containing that point. Fig. 3(a) shows the slice $z=-0.25$ of the Shepp-Logan phantom with the display interval $[0.95,1.05]$. The disk phantom consists of seven ellipsoids of half axes $0.7,0.7$ and 0.06 , placed 0.02 apart along the $z$-axis. All ellipsoids in the disk phantom are of density 1.0 . The slice $x=0$ of the disk phantom is shown in Fig. 3(b) with the display interval $[0,1]$.

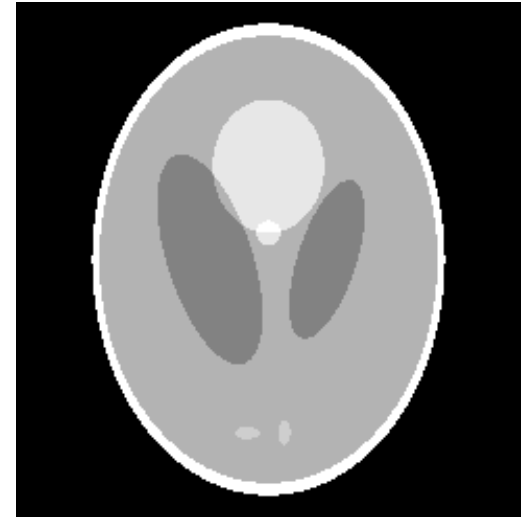

(a)

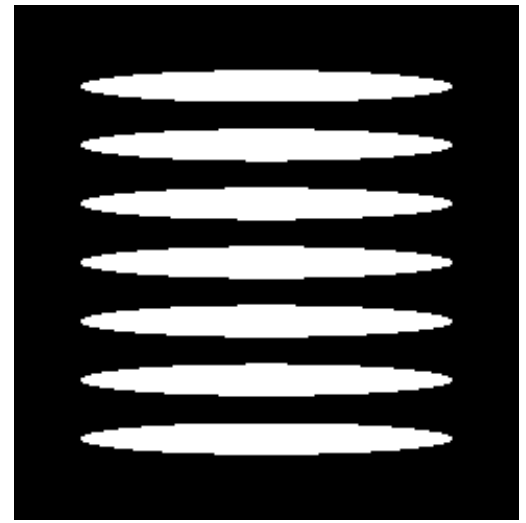

(b)

Fig. 3. Representative slices of the Shepp-Logan phantom and the disk phantom.

(a) Slice $z=-0.25$ of the Shepp-Logan phantom, and (b) slice $x=0$ of the disk phantom.

\subsection{Numerical results}

Fig. 4 shows the reconstruction images at $z=-0.25$ of the Shepp-Logan phantom using the generalized Feldkamp algorithm and the generalized Katsevich algorithm, respectively. The mean-squared-error between the original and reconstructed slices and the reconstruction time are listed in Table 3.

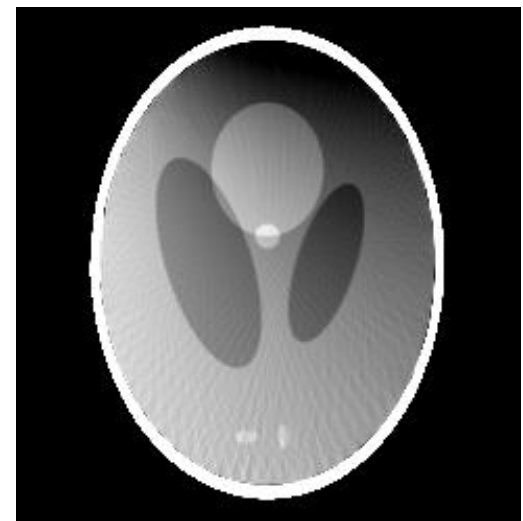

(a)

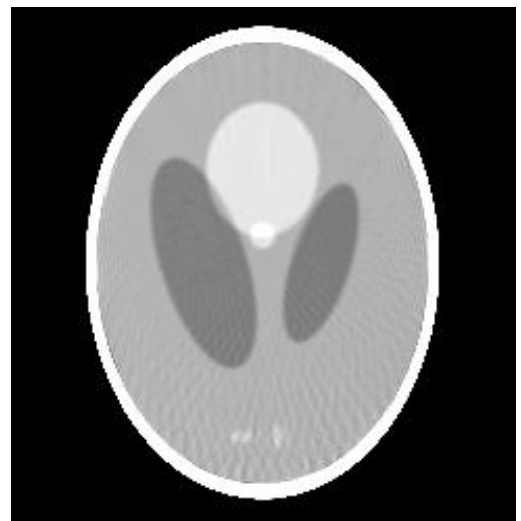

(b)

Fig. 4. Reconstructed slices of the Sheep-Logan phantom corresponding to Fig. 3(a).

(a) Generalized Feldkamp reconstruction, and (b) generalized Katsevich reconstruction.

Table 3. Experiment data from generalized Feldkamp and generalized Katsevich reconstructions.

\begin{tabular}{||c|c|c|c|c||}
\hline \multirow{2}{*}{} & \multicolumn{2}{|c|}{ Generalized Feldkamp algorithm } & \multicolumn{2}{c||}{ Generalized Katsevich algorithm } \\
\cline { 2 - 5 } & Fig. 4 (a) & Fig. 5(a) & Fig. 4(b) & Fig. 5(b) \\
\hline Mean-squared-error & 0.030 & 0.077 & 0.022 & 0.015 \\
\hline CPU time (min.) & 11.45 & 10.69 & 21.45 & 29.98 \\
\hline
\end{tabular}


It can be observed that the slice reconstructed by the generalized Katsevich algorithm contains little shading artifacts, while the generalized Feldkamp algorithm runs faster but induces a dark region in the top half part. As illustrated with the specified spiral locus, the generalized Feldkamp algorithm also requires a larger detector plate than the generalized Katsevich algorithm. This is because the full-scan based Feldkamp-type algorithm requires that cone-beam projections from "opposite" source positions within one spiral turn should overlap for full-scan based fan-beam reconstruction after the cone-beam data are corrected for their obliqueness ${ }^{4}$.

Experiments were also performed with the disk phantom to study the situation when the object contains a sharp gradient along the $z$-direction. Longitudinal cross-sections of the reconstructed disk phantom are shown in Fig. 5, corresponding to Fig. 3(b). It can be observed that with the disk phantom the generalized Feldkamp algorithm produces substantial artifacts between the ellipsoids, while the generalized Katsevich algorithm consistently gives excellent image quality.

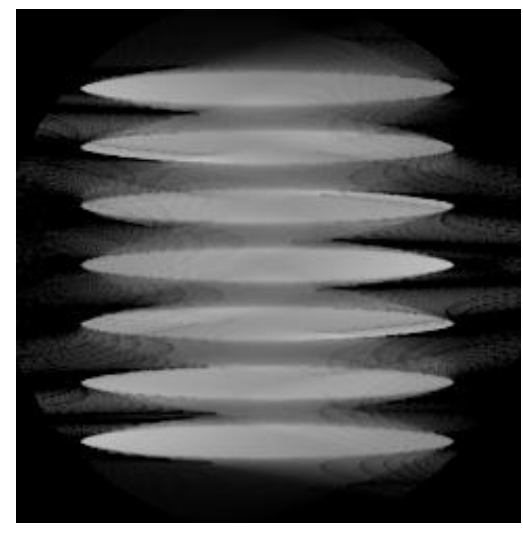

(a)

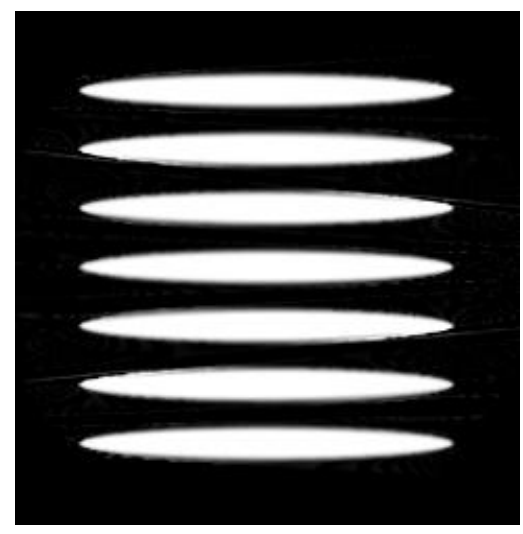

(b)

Fig. 5. Reconstructed slices of the disk phantom corresponding to Fig. 3(b).

(a) Generalized Feldkamp reconstruction, and (b)generalized Katsevich reconstruction.

To evaluate the noise characteristics of these two algorithms, we added Gaussian noise with standard deviation 1.0 to synthetic projection data. The noise level was evaluated by the mean-square difference between reconstruction with and without added noise. The noise measures with the generalized Feldkamp algorithm and the generalized Katsevich algorithm are 0.0035 and 0.0843 , respectively. This result indicates that the generalized Feldkamp algorithm is less sensitive to data noise, because it only requires high-pass filtering once while the generalized Katsevich algorithm requires high-pass filtering twice.

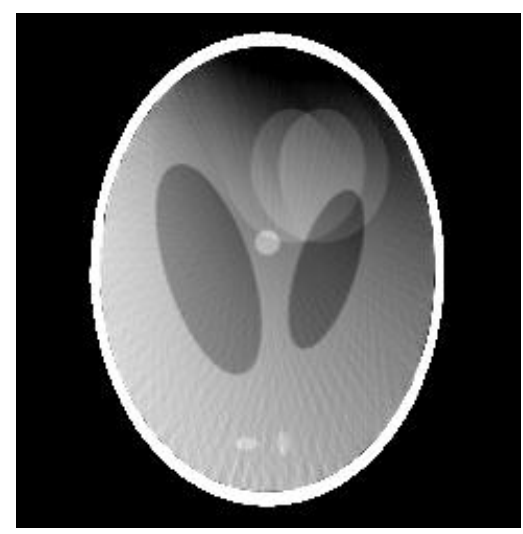

(a)

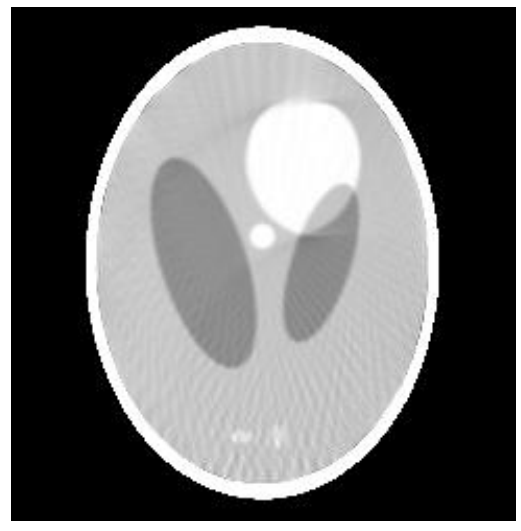

(b)

Fig. 6. Reconstructed slices of a dynamic Shepp-Logan phantom.

(a) Generalized Feldkamp reconstruction, and (b) generalized Katsevich reconstruction. 
Furthermore, we studied dynamic cone-beam reconstruction using these two algorithms. For that purpose, the largest bright ellipsoid in the Shepp-Logan phantom was moving uniformly along the horizontal direction. Fig. 6 shows generalized Feldkamp and generalized Katsevich reconstructions, respectively. There are much more motion artifacts in the generalized Feldkamp reconstruction than the generalized Katsevich counterpart, because we used the full-scan based Feldkamp-type algorithm. Clearly, the temporal resolution with the Feldkamp-type reconstruction can be easily improved using half-scan or PI-line based Feldkamp-type algorithms, but the noise performance may be accordingly compromised.

\section{DISCUSSIONS AND CONCLUSION}

The phantoms we used are still simplistic. It is highly desirable that the phantom be as realistic as possible in the CT simulation. Superquadrics ${ }^{10}$ are a family of $3 \mathrm{D}$ objects, which can be used to model a variety of objects. They have received a major attention in the fields of computer vision, computer graphics, and robotics, because of their compact form and representation capabilities. ${ }^{11}$ Recently we proposed an algorithm for the computation of the X-ray transform of a quite general type of superquadrics and demonstrated its utility with a superquadric-based human thorax phantom. ${ }^{12}$ Fig. 7 shows the slices of our human thorax phantom at $z=0$ and $y=0$. It can be observed that our phantom is more realistic than the popular phantom used in the literature. Currently, we are developing a dynamic mouse model based on superquadrics to test both approximate and exact cone-beam algorithms.

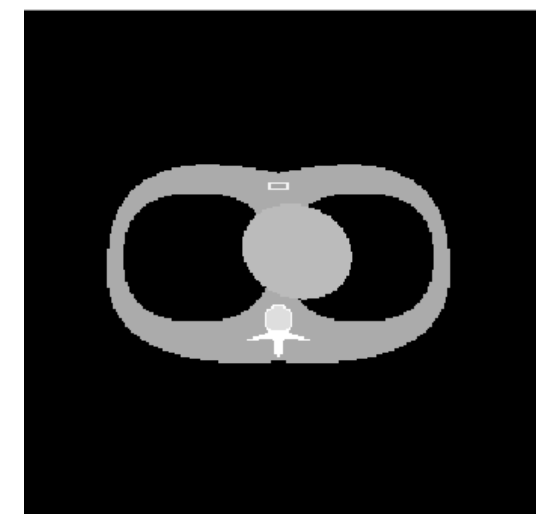

(a)

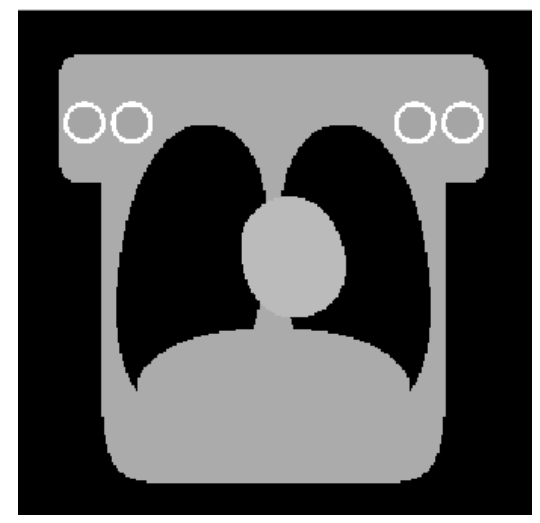

(b)

Fig. 7. Representative slices of a superquadrics-based human Thorax phantom.

(a) Slice $z=0$, and (b) slice $y=0$.

In conclusion, we have evaluated the generalized Feldkamp algorithm and the generalized Katsevich algorithm for EBMCT. Briefly speaking, the Feldkamp-type and Katsevich-type algorithms give comparable spatial resolution when the cone angle is not large (the spiral pitch is not large), and/or the longitudinal variation of the object is not dramatic. Generally speaking, the Feldkamp-type algorithm runs faster, is less sensitive to noise, more sensitive to motion, and produces more artifacts than the Katsevich-type algorithm. These results are considered a necessary and important step towards dynamic volumetric micro-CT of the small animals. Further comparative studies are underway to reveal their relative strengths and weaknesses systematically.

\section{ACKNOWLEDGEMENTS}

This work was partially supported by the NIH/NIBIB grants EB002667 and EB004287.

\section{REFERENCES}

1. G. Wang and Y. Ye, Nonstandard spiral cone-beam scanning methods, apparatus, and applications (Patent disclosure), 2003. 
2. G. Wang, C.R. Crawford, and W.A. Kalender, "Multirow detector and cone-beam spiral/helical CT", IEEE Trans. Med. Imaging, 19, 817-21, 2000.

3. L.A. Feldkamp, L.C. Davis, and J.W. Kress, "Practical cone-beam reconstruction", J. Opt. Soc. Am., A 1, 612-619, 1984.

4. G. Wang, T.H. Lin, P.C. Cheng, and D.M. Shinozaki, "A general cone-beam reconstruction algorithm”, IEEE Trans. Med. Imaging, 12, 486-496, 1993.

5. A. Katsevich, "An improved exact filtered backprojection algorithm for spiral computed tomography", Adv. Appl. Math., 32, 681-697, 2004.

6. Y. Ye, J. Zhu, and G. Wang, "Minimum Detection Windows, PI-line Existence and Uniqueness for Helical ConeBeam Scanning of Variable Pitch", Med. Phys. 31, 566-572, 2004.

7. Y. Ye, J. Zhu, and G. Wang, "Geometric Studies on Variable Radius Spiral Cone-Beam Scanning", Med. Phys. 31, 1473-1480, 2004.

8. H. Yu, Y. Ye, and G. Wang, "Katsevich-type algorithms for variable radius cone-beam CT", in SPIE Conference on Development in X-ray tomography IV, 2004, in this volume.

9. A.C. Kak, M. Slaney, Principles of Computerized Tomographic Imaging, SIAM, 2001.

10. A. H. Barr, "Superquadrics and angel preserving transformations", IEEE Computer Graphics and Applications, 1, $11-23,1981$.

11. A. Jaklic, A. Leonardis, F. Solina, Segmentation and Recovery of Superquadrics, Kluwer Academic Publishers, 2000.

12. J. Zhu, S. Zhao, Y. Ye and G. Wang, "Superquadric-based modeling for computed tomography simulation", submitted.

*ge-wang@uiowa.edu 